\title{
Hepatectomy without endotracheal general anesthesia: a safe procedure?
}

\author{
Hovaguimian, Frederique ; Beck-Schimmer, Beatrice ; Clavien, Pierre-Alain ; Schwartz, David E
}

DOI: https://doi.org/10.1016/j.jamcollsurg.2013.11.009

Posted at the Zurich Open Repository and Archive, University of Zurich ZORA URL: https://doi.org/10.5167/uzh-99695

Journal Article

Originally published at:

Hovaguimian, Frederique; Beck-Schimmer, Beatrice; Clavien, Pierre-Alain; Schwartz, David E (2014). Hepatectomy without endotracheal general anesthesia: a safe procedure? Journal of the American College of Surgeons, 218(3):499-500.

DOI: https://doi.org/10.1016/j.jamcollsurg.2013.11.009 


\section{Dear Editor,}

We would like to refer to the article entitled "First Report of Hepatectomy without Endotracheal General Anesthesia" from Yamamoto et al., published May 2013.

The authors report on 10 patients undergoing liver resection under epidural anesthesia (EDA) with conscious sedation (a procedure without intubation) assessing thereby five endpoints: mortality rate, surgical time, blood loss, amount of applied local anesthetic and central venous pressure. The results section provides endpoints values, together with a variety of perioperative data, mainly complication rates. The authors conclude that this innovative anesthetic management can be considered as a valuable alternative to general anesthesia (GA).

We commend our colleagues for their initiative to bring new perspectives in our clinical practice. There is nowadays an increasing demand for high quality of care and innovative projects may improve clinical performance and patient safety. However, some of the conclusions provided in this article are questionable.

First, the methodology used in this report raises serious concerns. Developed by expert committees, internationally recognized safety indicators are nowadays widely used when assessing quality of care ${ }^{1}$ especially in the perioperative setting. 'Mortality' given without any time range, is a poor safety indicator in anesthesia, not only because of its low occurrence, ${ }^{3}$ but also because of the lack of standardized definition. ${ }^{4}$ While 'blood transfusion requirement' provides reliable information, the 'amount of bleeding' does not. Moreover, how the authors determined the rate of postoperative complications remains obscure: there is no information regarding the criteria used to define complications ('pneumonia', for example, is a very imprecise entity), and a description of the process used to screen patients for complications is lacking.

Secondly, the rationale behind this article remains unclear. An increasing number of publications provide evidence for improved pulmonary function when using EDA, ${ }^{5}$ and vulnerable patients such as severe chronic obstructive pulmonary diseases patients could certainly benefit from this protective effect. However, the advantage of EDA for upper abdominal procedures has been established exclusively in procedure combining EDA with GA. Besides providing optimal conditions for the surgeon (relaxation and controlled ventilation), GA ensures appropriate safety procedure in settings where the limits of 'conscious sedation' are rapidly reached. It is indeed hard to believe that patients deemed unfit for GA would have a better outcome after 5 hours of subdiaphragmatic surgery in supine position with substantial intravenous doses of benzodiazepines. Besides discomfort and distress, the incidence of hypoventilation, hypoxemia, or aspiration would certainly reach worrying values. 
Finally, this article raises some ethical concerns. A research hypothesis should be supported by convincing scientific evidence warranting that study patients are not exposed to unnecessary risks: in abdominal surgery, formal evidence that 'conscious sedation' is regarded as a safer procedure than GA has not been described. Moreover, although some patients were eligible for a less invasive procedure (laparoscopy), they underwent a more aggressive surgery (open resection), only for the purpose of the study. Clinical research allows certainly for innovation, but designing a research protocol should always include basic ethical considerations and an assessment of the risk-benefit ratio. ${ }^{6}$

\section{REFERENCES}

1. Benn J, Arnold G, Wei I, Riley C, Aleva F. Using quality indicators in anaesthesia: feeding back data to improve care. Br J Anaesth. Jul 2012;109(1):80-91.

2. Haller G, Stoelwinder J, Myles PS, McNeil J. Quality and safety indicators in anesthesia: a systematic review. Anesthesiology. May 2009;110(5):1158-1175.

3. Staender SE, Mahajan RP. Anesthesia and patient safety: have we reached our limits? Curr Opin Anaesthesiol. Jun 2011;24(3):349-353.

4. Merry AF. An overview of quality and safety in health care. Can J Anaesth. Feb 2013;60(2):101-104.

5. Popping DM, Elia N, Marret E, Remy C, Tramer MR. Protective effects of epidural analgesia on pulmonary complications after abdominal and thoracic surgery: a metaanalysis. Arch Surg. Oct 2008;143(10):990-999; discussion 1000.

6. Emanuel EJ, Wendler D, Grady C. What makes clinical research ethical? JAMA. May 24-31 2000;283(20):2701-2711.

Frederique Hovaguimian, MD, and Beatrice Beck-Schimmer, MD, Institute of Anesthesiology, University Hospital Zurich, Zurich, Switzerland

Pierre-Alain Clavien, MD, Department of Surgery, University Hospital Zurich, Zurich, Switzerland David E. Schwartz, MD, Department of Anesthesiology, University of Illinois at Chicago, College of Medicine, Chicago 